\title{
PSR B 1706-44 and the SNR G 343.1-2.3 as the remnants of a cavity supernova explosion
}

\author{
D. C.-J. Bock ${ }^{1}$ and V. V. Gvaramadze $\mathrm{B}^{2,3,4, \star}$ \\ 1 Radio Astronomy Laboratory, University of California, Berkeley, CA 94720, USA \\ 2 Sternberg State Astronomical Institute, Moscow State University, Universitetskij Pr. 13, Moscow, 119992, Russia \\ 3 E.K.Kharadze Abastumani Astrophysical Observatory, Georgian Academy of Sciences, A.Kazbegi ave. 2-a, Tbilisi, 380060 , \\ Georgia \\ 4 Abdus Salam International Centre for Theoretical Physics, Strada Costiera 11, PO Box 586, 34100 Trieste, Italy
}

Received 11 April 2002 / Accepted 1 August 2002

\begin{abstract}
The possible association of the supernova remnant (SNR) G 343.1-2.3 with the pulsar PSR B 1706-44 (superposed on the arclike "shell" of the SNR) has been questioned by some authors on the basis of an inconsistency between the implied and measured (scintillation) transverse velocities of the pulsar, the absence of any apparent interaction between the pulsar and the SNR's "shell", and some other indirect arguments. We suggest, however, that this association could be real if both objects are the remnants of a supernova (SN) which exploded within a mushroom-like cavity (created by the SN progenitor wind breaking out of the parent molecular cloud). This suggestion implies that the actual shape of the SNR's shell is similar to that of the well-known SNR VRO 42.05.01 and that the observed bright arc corresponds to the "half" of the SNR located inside the cloud. We report the discovery in archival radio data of an extended ragged radio arc to the southeast of the bright arc which we interpret as the "half" of the SN blast wave expanding in the intercloud medium.
\end{abstract}

Key words. stars: neutron - stars: pulsars: individual: PSR B 1706-44 - ISM: bubbles - ISM: individual objects: G 343.1-2.3 - ISM: supernova remnants

\section{Introduction}

The pulsar PSR B 1706-44 (Johnston et al. 1992) is superposed on an incomplete arc of radio emission (McAdam et al. 1993). McAdam et al. interpreted this arc as a shell-type supernova remnant (SNR), named G 343.1-2.3, and suggested that the SNR is physically associated with PSR B 1706-44. This suggestion was questioned by Frail et al. (1994a) and Nicastro et al. (1996; see, however, Dodson et al. 2001). Usually, a particular claimed pulsar/SNR association is considered reliable if the following five criteria are fulfilled (e.g. Kaspi 1996):

1. agreement of independent distance estimates for pulsar and SNR;

2. agreement of independent age estimates for pulsar and SNR;

3. consistence of the implied pulsar transverse velocity (i.e. the velocity inferred by the displacement of the pulsar from the geometrical centre of the associated SNR) with the measured (proper motion and/or scintillation) velocity;

4. existence of any sign of interaction between the pulsar and the SNR;

Send offprint requests to: D. C.-J. Bock, e-mail: dbock@astro.berkeley.edu

* Address for correspondence: Krasin str. 19, ap. 81, Moscow, 123056, Russia (e-mail: vgvaram@sai .msu.ru)
5. "correct" (inferred or measured) orientation of the vector of pulsar transverse velocity (it is assumed that this vector should be pointed away from the geometrical centre of the associated SNR).

Although the distance and age estimates for PSR B 1706-44 and G343.1-2.3 are in reasonable agreement, the implied transverse velocity is at least an order of magnitude larger than the scintillation velocity calculated by Nicastro et al. (1996). This inconsistency along with the absence of any apparent interaction between the pulsar and the SNR constitute the two main arguments against the physical association between these two objects (Frail et al. 1994a; Nicastro et al. 1996). The fifth criterion is not applied to the system, since the direction of the pulsar proper motion is still unknown (cf. Giacani et al. 2001 with Frail et al. 1994a; see also Sect. 3.5). Additional (indirect) arguments against the association are based on Gaensler \& Johnston's (1995) statistical study, which suggests that young pulsars cannot overrun their parent SNR shells (Nicastro et al. 1996; see however Arzoumanian et al. 2002) and on the large extent of the "halo" around the pulsar (Frail et al. 1994a).

In this paper we show how the existing observational data on PSR B 1706-44 and G343.1-2.3 can be interpreted in favour of their physical association (Sect. 2) and discuss the criteria for evaluating the reliability of pulsar/SNR associations 
as applied to this system (Sect. 3). The main suggestion of the paper is that the association between PSR B 1706-44 and the SNR G 343.1-2.3 could be real if both objects are the remnants of a SN which exploded within a mushroom-like cavity created by the SN progenitor wind breaking out of the parent molecular cloud (Sect. 2.2). This suggestion implies that in addition to the known bright "half" of the SNR G 343.1-2.3 there should exist a more extended and weaker component, so that the actual shape of G343.1-2.3 is similar to that of the well-known SNR VRO 42.05.01. It is remarkable that the $2.4 \mathrm{GHz}$ Parkes Survey of Duncan et al. (1995) shows the existence of such an extended component.

\section{The SNR G 343.1-2.3}

Before discussing the criteria for evaluating the reliability of the association between PSR B 1706-44 and the SNR G 343.1-2.3 we review the observational data on this system and propose a scenario for its formation.

\subsection{Observational data}

SNR G 343.1-2.3 was discovered by McAdam et al. (1993). Their 843 MHz image of G 343.1-2.3 shows a well-defined arc (a half-ellipse) of radio emission with the brightest (northern) part closest to the Galactic plane. The maximum extent of the arc is about $40^{\prime}$. A VLA image of the SNR obtained by Frail et al. (1994a) shows the existence of weak, diffuse emission both inside and outside the bright arc. This emission fills a region similar to and about two times more extended than the bright arc (Dodson et al. 2001; see also Duncan et al. 1995 and Fig. 2). The ROSAT observations of the field around PSR B 1706-44 do not reveal any sign of correlation between the soft $(0.1-2.4 \mathrm{keV})$ diffuse $\mathrm{X}$-ray emission and the radio emission of the SNR (Becker et al. 1995). There are no reported optical observations of the SNR. PSR B 1706-44 is superposed on the outside edge of the bright radio arc and there are no morphological signatures of interaction between them. The pulsar appears to be surrounded by a radio nebula of about $3^{\prime}$ in size (Giacani et al. 2001; cf. Frail et al. 1994a). Giacani et al. suggest that this nebula is powered by the pulsar, on the basis of the nebula's flat radio spectral index $(\simeq 0.3)$ and the high mean fractional polarization $(\simeq 20 \%)$ of its radio emission.

\subsection{A scenario for the origin of the system PSR B 1706-44/G 343.1-2.3}

We suggest that the SNR G343.1-2.3 is the result of an off-centred cavity SN explosion. Figure 1 schematically depicts a scenario for its origin. A massive star (the progenitor of the $\mathrm{SN}$ ) ends its evolution within a mushroom-like cavity formed by the SN progenitor wind breaking out of the parent molecular cloud and expanding into an inter-cloud medium of much less density. The proper motion of the progenitor star results in a considerable offset of the SN explosion site from the geometrical centre of the semi-spherical cavity created inside the cloud; we suggest that the SN exploded outside the cloud.
The subsequent interaction of the SN blast wave with the reprocessed ambient medium determines the structure of the resulting SNR (e.g. Ciotti \& D'Ercole 1989; Chevalier \& Liang 1989; Franco et al. 1991), which acquires a form reminiscent of the well-known SNR VRO 42.05.01 (G 166.0+4.3) ${ }^{1}$.

We speculate that the wind-blown cavity formed inside the cloud was surrounded by a shell of mass less than some critical value (for spherically-symmetric shells this value is about 50 times the mass of the SN ejecta; e.g. Franco et al. 1991), so that the SN blast wave was able to overrun the shell to propagate further into the unperturbed gas of the cloud, leaving behind the reaccelerated and gradually broadening turbulent shell (Franco et al. 1991). We suggest that the bright arc discovered by McAdam et al. corresponds to the shocked former winddriven shell and that the diffuse radio emission seen by Frail et al. and Dodson et al. comes from the "half" of the SN blast wave propagating into the cloud (see Fig. 1). These two components correspond to the bright arclike structure in the low resolution image of G343.1-2.3 (Fig. 2). Under this scenario a more extended component of the SNR should exist to the southeast of the known bright structure. This would correspond to the "half" of the SN blast wave expanding in the inter-cloud medium.

We have found such a feature in the $2.4 \mathrm{GHz}$ Parkes Survey of the Galactic Plane (Duncan et al. 1995; Fig. 2) - an extended arc of diffuse emission stretched from $(l=345, b=-2.5)$ to $(l=342, b=-4.5)$. Its location in the "proper" place and its symmetry with respect to the bright arc of G343.1-2.3 suggest that it could be physically related to this SNR. We note that the ragged appearance of the extended component could result from the development of a Rayleigh-Taylor instability caused by the impact of the SN blast wave with the wall of the low-density cavity created in the inter-cloud medium by the SN progenitor wind breaking out of the cloud (cf. Gvaramadze 1999a). Note however that Duncan et al. consider this feature to be a part of a larger complex of filaments. The association between the extended arc and the SNR G343.1-2.3 may be further investigated by detailed $\mathrm{H}_{\mathrm{I}}$ mapping in the vicinity of the arc or by combining new continuum interferometric observations with the existing single dish maps of the region.

\section{Reliability of the association between the pulsar PSR B 1706-44 and the SNR G 343.1-2.3}

We now discuss the criteria for evaluating the reliability of pulsar/SNR associations (Sect. 1) as applied to the system PSR B 1706-44/G 343.1-2.3. It is obvious that the first two criteria should be fulfilled for any proposed pulsar/SNR association. Application of the third and fifth ones for evaluating

\footnotetext{
${ }^{1}$ It is believed that the unusual appearance of VRO 42.05 .01 is due to the breaking out of the SN blast wave into a hot, low-density tunnel, whose origin is the result of an amalgamation of cavities created by one or more SN explosions or stellar winds (e.g. Pineault et al. 1987). We suggest that an alternative explanation of the origin of SNRs of this type (another example is the SNR G 350.0-3.0) is a SN explosion inside a (mushroom-like) wind-driven cavity created near the edge of a molecular cloud. Numerical simulations of this situation would be highly desirable.
} 


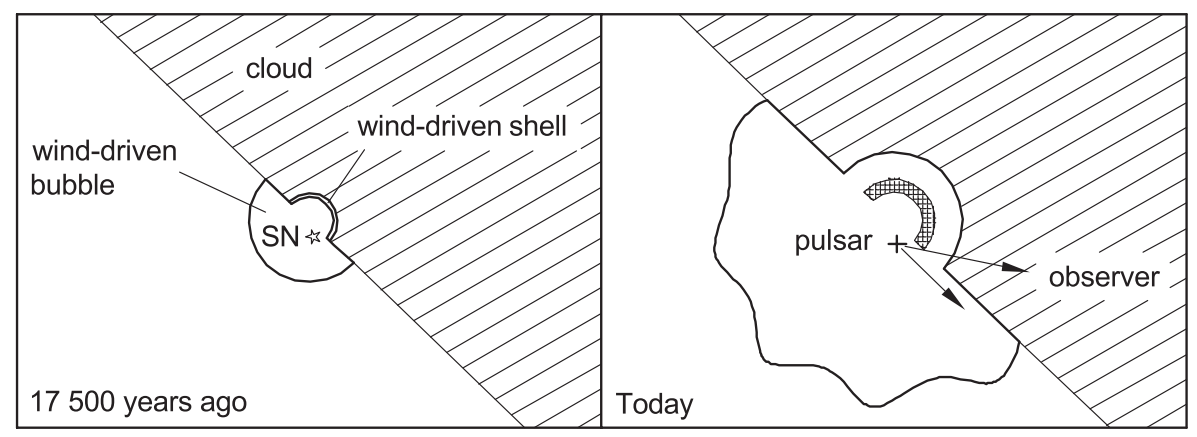

Fig. 1. Schematic of the proposed origin of G 343.1-2.3 (not to scale).

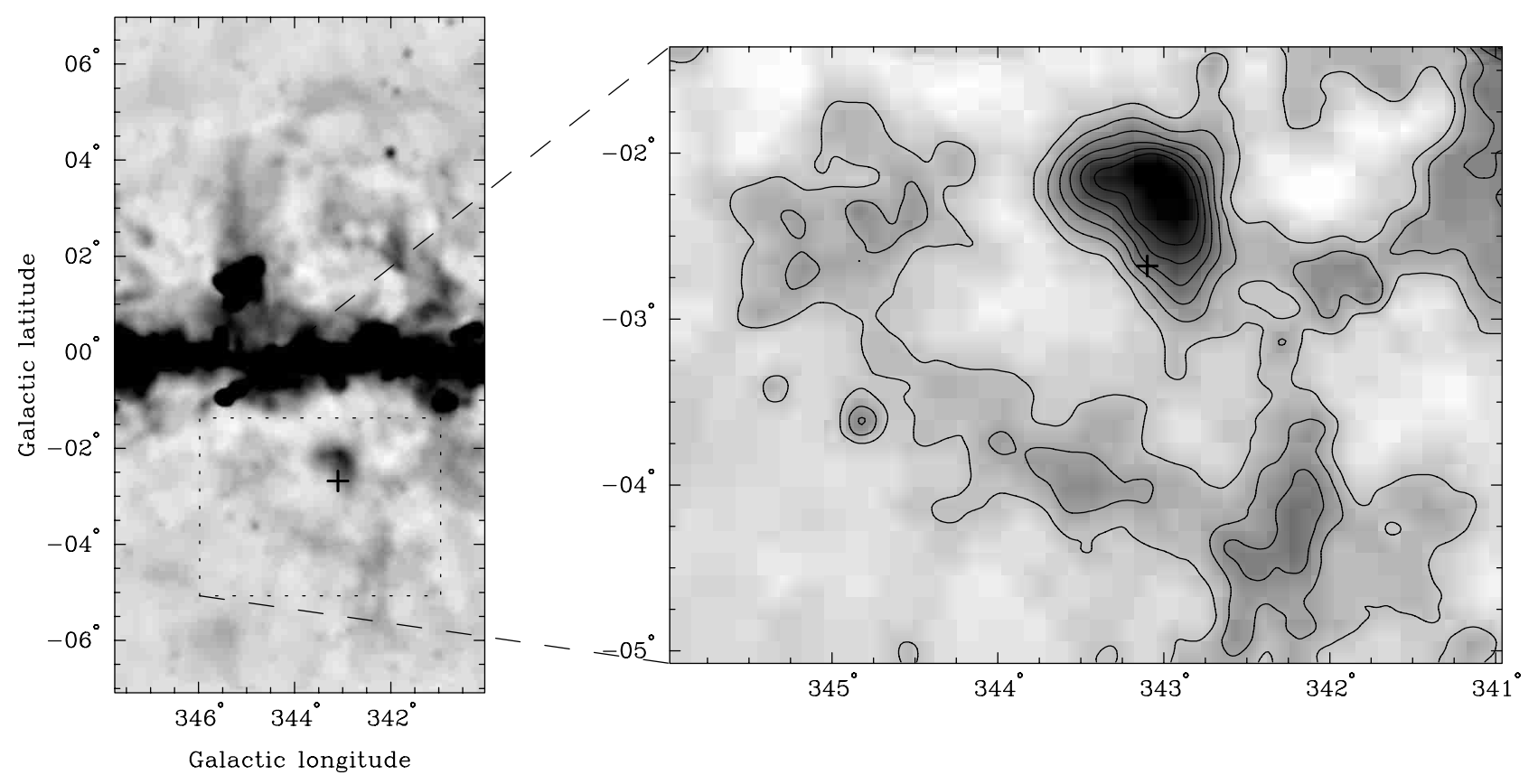

Fig. 2. 2.4 GHz image of G 343.1-2.3 (Duncan et al. 1995). A cross marks the position of the pulsar.

proposed associations is not so straightforward, since they are based on the assumption that the SN explosion site coincides with the geometrical centre of the SNR. This assumption could be erroneous in the case of a density-stratified interstellar medium (e.g. Lozinskaya 1992; Frail et al. 1994b) or in the case of a cavity SN explosion (Gvaramadze 2002). A mechanical application of these two criteria could result in the rejection of genuine associations (Gvaramadze 2002). The fourth criterion should be considered for those claimed associations where the pulsar is located not far (at least in projection) from the SNR's shell. To some extent this situation takes place in the case considered in this paper - the pulsar is superposed on the bright radio arc.

\subsection{The distance estimates}

The most reliable distance estimate for PSR B 1706-44 was derived by Koribalski et al. (1995) using a kinematic method. This estimate ranges from $2.4 \pm 0.6$ to $3.2 \pm 0.4 \mathrm{kpc}$, in quite reasonable agreement with the dispersion measure distance of $1.8 \pm 0.5 \mathrm{kpc}$ derived from the Taylor \& Cordes' (1993) model for the Galactic electron density distribution, given the uncertainties inherent in both methods. The kinematic distance measured by Koribalski et al. also agrees with the distance estimates for G 343.1-2.3 obtained by McAdam et al. (1993) and Frail et al. (1994a). But the latter estimates are very uncertain, since they are based on the highly controversial empirical relationship between the observed surface brightness of SNRs and their linear diameters (see e.g. Green 1991; but see also Huang et al. 1994). These estimates would be even less certain if our scenario for the origin of the SNR is correct. In what follows we assume a distance to the pulsar (and the SNR) of $(1.8+2.4) / 2=2.1 \mathrm{kpc}$.

\subsection{The age estimates}

The characteristic age of PSR B 1706-44 is $\tau=P /(n-1) \dot{P}$, where $P$ is the spin period of the pulsar, $\dot{P}$ is the period derivative, and $n$ is the braking index. For $P=0.102 \mathrm{~s}, \dot{P}=9.3 \times$ $10^{-14} \mathrm{~s} \mathrm{~s}^{-1}$ (Johnston et al. 1992), and assuming that $n=3$, one has $\tau \simeq 17500 \mathrm{yr}$. This age can be compared with the age estimate for G 343.1-2.3 of 5000-6000 yr, derived (McAdam et al. 1993; Nicastro et al. 1996) on the basis of the Sedov-Taylor 
solution (i.e. from the diameter-age relationship, e.g. Clark \& Caswell 1976). But the diameter-age relationship cannot be applied to SNRs originating from cavity SN explosions. In what follows we assume that the true age of the pulsar is equal to $\tau$ and that the SNR is as old as the pulsar. We realize, however, that the system could be younger (if the pulsar was born with a spin period close to the current one; in this case $\tau$ overestimates the true age of the pulsar) or older (see Sect. 3.3.1), but the actual age of the system is not fundamental to the results presented in this paper.

\subsection{The pulsar velocity}

\subsubsection{The implied velocity}

The implied pulsar transverse velocity, i.e. the velocity inferred from the angular displacement of PSR B 1706-44 from the geometrical centre of the bright arc, about $20^{\prime}$ north of the pulsar, is

$V_{\mathrm{imp}} \simeq 700 \theta_{20} D_{2.1} \tau_{17.5}^{-1} \mathrm{~km} \mathrm{~s}^{-1}$

where $\theta_{20}$ is the angular displacement in units of $20^{\prime}, D_{2.1}$ is the distance to the pulsar in units of $2.1 \mathrm{kpc}$, and $\tau_{17.5}$ is the characteristic age of the pulsar in units of $17.5 \mathrm{kyr}$. In principle this high transverse velocity is not impossible. It could be even higher if the true age of the pulsar is less than $\tau$ (see Sect. 3.2). However it can be reduced if one assumes that the true age of the pulsar is much larger than the characteristic one (e.g. due to a low value of the pulsar braking index (e.g. Camilo 1996) or due to a secular (e.g. Blandford \& Romani 1988) or shortterm (e.g. Gvaramadze 1999b, 2001a) increase of the braking torque). The implied velocity can also be reduced if the true SN explosion site is offset from the geometrical centre of the SNR. Such offsets naturally arise if the SN exploded in a density-stratified medium (e.g. Lozinskaya 1992; Frail et al. 1994 b) or inside a cavity created by the wind of the moving SN progenitor star (Gvaramadze 2002). We favour the last possibility (Sect. 2.2) and suggest that the pulsar transverse velocity could be less than the implied one (see Sect. 3.3.2).

The implied transverse velocity should be compared with the measured one. Nicastro et al. (1996) derived the pulsar velocity from the scintillation measurements and found that it is anomalously low, about twenty times less than $V_{\text {imp. They }}$ used this inconsistency to suggest that the pulsar did not originate from the apparent centre of SNR, and that the pulsar and SNR are not associated. We agree with their first suggestion (see Sect. 2.2) and therefore believe that the implied velocity can be reduced. On the other hand we have found (Sect. 3.3.2) that if the turbulent material of the reaccelerated former winddriven shell (the bright arc of SNR G 343.1-2.3) is responsible for nearly all the scattering of PSR B 1706-44, then the pulsar transverse velocity can be as large as the transverse velocity of the portion of the arc projected on the pulsar. If so, one can show that the pulsar (transverse) velocity should indeed be less than $V_{\text {imp }}$, though it can be much larger than that calculated by Nicastro et al. (1996).

\subsubsection{The scintillation velocity}

It is known that pulsar velocities derived from scintillation measurements show good correlation with proper-motionderived ones (e.g. Gupta 1995). However, scintillation velocities are derived under several assumptions, some of which are not necessarily suitable for individual pulsars. It is usually assumed that the scattering material is homogeneously distributed along the line of sight and that its transverse velocity is negligible. These assumptions could be erroneous if a considerable fraction of the scintillations is due to a localized region of enhanced scattering existing along the line of sight in addition to the distributed scattering medium and if the transverse velocity of this region is nonzero (e.g. Cordes \& Rickett 1998). In this case the scintillation velocity of the pulsar is not equal to the proper motion velocity. In the following we assume that the turbulent material associated with the bright arc of the SNR G 343.1-2.3 is the main scatterer of PSR B 1706-44.

The scintillation velocity for an asymmetrically placed thin scattering screen is (e.g. Gupta 1995):

$V_{\mathrm{iss}}=3.85 \times 10^{4} \frac{\left(v_{\mathrm{d}, \mathrm{MHz}} D_{\mathrm{kpc}} x\right)^{1 / 2}}{f_{\mathrm{GHz}} t_{\mathrm{d}}} \mathrm{km} \mathrm{s}^{-1}$,

where $v_{\mathrm{d}, \mathrm{MHz}}$ and $t_{\mathrm{d}}$ are the scintillation bandwidth and the time-scale measured respectively in $\mathrm{MHz}$ and seconds, $D_{\mathrm{kpc}}$ is the distance from observer to pulsar in kpc, $x=D_{\mathrm{o}} / D_{\mathrm{p}}$, $D_{\mathrm{o}}$ and $D_{\mathrm{p}}$ are the distances from observer to screen and from screen to pulsar, and $f_{\mathrm{GHz}}$ is the frequency of observation in units of GHz. Note that the numerical coefficient in Eq. (1) is about three times larger than that used by Nicastro et al. (1996; see, however, Johnston et al. 1998). With $v_{\mathrm{d}, \mathrm{MHz}}=15$, $t_{\mathrm{d}}=2287, f_{\mathrm{GHz}}=1.52$, and assuming that $x=1$ (Nicastro et al. 1996), one has for PSR B 1706-44 that $V_{\text {iss }}=62 D_{2.1}^{1 / 2} \mathrm{~km} \mathrm{~s}^{-1}$. Although this value is few times larger than that calculated by Nicastro et al. (1996), it is still at the lower limit of the pulsar velocity distribution (e.g. Blaauw \& Ramachandran 1998; Lorimer et al. 1997). However, the pulsar velocity estimate can be further increased if one takes into account the transverse velocity of the scattering screen.

In the observer's reference frame the scintillation velocity is connected with the pulsar (transverse or proper motion) velocity $V_{\mathrm{p}}$ by the following relationship (Gvaramadze 2001b; cf. Gupta et al. 1994; Cordes \& Rickett 1998):

$$
\begin{aligned}
V_{\mathrm{iss}}= & {\left[x^{2} V_{\mathrm{p}}^{2}-2 x(1+x) V_{\mathrm{p}} V_{\mathrm{scr}, \|}\right.} \\
& \left.+(1+x)^{2} V_{\mathrm{scr}, \|}^{2}+(1+x)^{2} V_{\mathrm{scr}, \perp}^{2}\right]^{1 / 2},
\end{aligned}
$$

where $V_{\text {scr,\| }}$ and $V_{\text {scr, } \perp}$ are the components of the transverse velocity of the screen, correspondingly, parallel and perpendicular to the vector of the pulsar proper motion velocity. In (2) we neglected for simplicity contributions from the differential Galactic rotation and the Earth's orbital motion around the Sun. If $V_{\text {scr }}=0$ (as assumed by Nicastro et al. 1996), one has $V_{\mathrm{p}}=V_{\text {iss }} / x \simeq 62 x^{-1 / 2} D_{2.1}^{1 / 2} \mathrm{~km} \mathrm{~s}^{-1} \simeq 0$ (note that $\left.x \sim 100\right)$. But, if $V_{\mathrm{scr}} \neq 0$, one can solve Eq. (2) for $V_{\mathrm{p}}$ :

$V_{\mathrm{p}} \simeq V_{\mathrm{scr}, \|} \pm\left(V_{\mathrm{iss}}^{2} / x^{2}-V_{\mathrm{scr}, \perp}^{2}\right)^{1 / 2}$. 
This solution is physically meaningful only for $V_{\mathrm{scr}, \perp} \leq$ $V_{\text {iss }} / x(\simeq 0)$, i.e. if the pulsar moves in the same direction and with nearly the same (transverse) velocity as does the part of the SNR responsible for the scattering of the pulsar: $V_{\mathrm{p}} \simeq V_{\text {scr,\|. }}$.

Although the existing observational data do not allow us to estimate the expansion velocity of the bright arc, we can constrain it by setting an upper limit on the expansion velocity of the "half" of the SN blast wave propagating inside the cloud. This constraint can be derived from the non-detection of the soft $(0.1-2.4 \mathrm{keV}) \mathrm{X}$-ray emission from the SNR. For the column density towards the SNR of $\simeq 2-5 \times 10^{21} \mathrm{~cm}^{-2}$ (Becker et al. 1995), the interstellar medium transmits essentially no $\mathrm{X}$-ray emission with energies below $0.3 \mathrm{keV}$ (e.g. Gorenstein $\&$ Tucker 1976), therefore the expansion velocity of the blast wave is less than $\simeq 500 \mathrm{~km} \mathrm{~s}^{-1}$. On the other hand, it is obvious that the former wind-driven shell lagging behind the blast wave (whose angular extent is about two times larger) expands at least two times more slowly. These arguments show that if the bright arc is the main scatterer of the pulsar's radio emission, then the pulsar transverse velocity should indeed be less than $V_{\text {imp }}$, though it could be as large as $200 \mathrm{~km} \mathrm{~s}^{-1}$.

\subsection{Interaction between the pulsar and the SNR}

We mentioned above that despite the apparent proximity of PSR B 1706-44 to the bright arc of the SNR G 343.1-2.3 there are no morphological signatures of interaction between these two objects. This has given some authors a basis to question their association (Frail et al. 1994a; see also Nicastro et al. 1996). But this inconsistency can be easily removed if the SN exploded within a mushroom-like wind-driven cavity (Sect. 2.2).

\subsection{Orientation of the pulsar proper motion vector}

The proper motion vectors of neutron stars born in off-centred cavity SN explosions can be oriented arbitrarily with respect to the geometric centres of the associated SNRs (Gvaramadze 2002); they can even be directed towards the geometric centres of the SNRs ${ }^{2}$ ! In Sect. 3.3 we showed that if the scintillations of PSR B 1706-44 are due mostly to the scattering in the turbulent material associated with the bright arc of SNR G 343.1-2.3, then the pulsar proper motion should be parallel to the expansion velocity of this material, i.e. the pulsar should move from the northeast to the southwest; this should be tested observationally.

\subsection{On statistical studies of pulsar/SNR associations}

Now we discuss the statistical argument against the association between PSR B 1706-44 and the SNR G 343.1-2.3 mentioned by Nicastro et al. (1996). This argument is based on the result of a statistical study of pulsar/SNR associations by Gaensler \& Johnston (1995), which suggests that young (<25000 yr)

\footnotetext{
${ }^{2}$ Perhaps exactly this situation takes place in the case of the pulsar PSR B 0656+14, which is moving approximately towards the centre of the SNR Monogem Ring (Thompson \& Córdova 1994).
}

pulsars cannot overrun their parent SNR shells (we recall that the spin-down age of PSR B 1706-44 is $\simeq 17500 \mathrm{yr}$ ). Although it is now clear that PSR B 1706-44 is located (at least in projection) well within the SNR G 343.1-2.3 (Dodson et al. 2001), it should be mentioned that Gaensler \& Johnston (1995) did not consider two very important effects: modification of the ambient medium by the ionizing emission and stellar wind of massive stars (the progenitors of most $\mathrm{SNe}$ ), and the proper motion of SN progenitor stars. Taking into account these two effects allows it to be shown that even a young pulsar moving with a moderate velocity $\left(\simeq 200 \mathrm{~km} \mathrm{~s}^{-1}\right)$ is able to escape the SNR's shell, provided that it was born not far from the edge of the wind-driven bubble (Gvaramadze 2002). Alternatively, the apparent location of a pulsar on the edge of a SNR's shell can be due simply to the effect of projection in non-sphericallysymmetric SNRs (see Fig. 1).

\subsection{On the wind nebula around PSR B 1706-44}

The large angular extent of the pulsar wind nebula was used by Frail et al. (1994a) to consider an association between the pulsar and the SNR unlikely. Assuming that the pulsar moves (with a velocity of $670 \mathrm{~km} \mathrm{~s}^{-1}$ ) through the interstellar medium (of number density $1 \mathrm{~cm}^{-3}$ ) and that the pulsar wind nebula is confined by the ram pressure of the ambient medium, they found that the characteristic radius of the nebula, $r_{\mathrm{PWN}}$, should be about two orders of magnitude less than observed. Based on this argument Frail et al. suggested that G 343.1-2.3 could be a background object, while PSR B 1706-44 could be a lowvelocity pulsar. We agree with their last suggestion, but propose that the pulsar wind nebula is instead confined by the interaction with the hot, tenuous material which fills the interior of the SNR:

$\frac{|\dot{E}|}{4 \pi c r_{\mathrm{PWN}}^{2}} \simeq n\left(2 k T+\mu m_{\mathrm{H}} v_{\mathrm{p}}^{2}\right)$,

where $|\dot{E}|=3.4 \times 10^{36} \mathrm{ergs} \mathrm{s}^{-1}$ is the pulsar spin-down luminosity, $c$ is the speed of light, $n$ and $T$ are, respectively, the number density and the temperature inside the SNR, $k$ is the Boltzmann constant, $\mu=1.3$ is the mean molecular weight, $m_{\mathrm{H}}$ is the mass of a hydrogen atom, and $v_{\mathrm{p}}$ is the full (i.e. three-dimensional) pulsar velocity. For $r_{\mathrm{PWN}} \simeq 0.9 D_{2.1}^{-1} \mathrm{pc}$ and $v_{\mathrm{p}} \simeq 200 \mathrm{~km} \mathrm{~s}^{-1}$, and assuming that $T \simeq 10^{7} \mathrm{~K}$, one has from Eq. (3) a quite reasonable estimate for the number density, $n=3.2 \times 10^{-4} \mathrm{~cm}^{-3}$. Note that this estimate could be somewhat altered should the pulsar velocity have a significant radial component (a case not constrained by the considerations of this paper). For example, if $v_{\mathrm{p}}=500 \mathrm{~km} \mathrm{~s}^{-1}$, then one has $n=1.4 \times 10^{-4} \mathrm{~cm}^{-3}$ (i.e. also a reasonable value).

\section{Conclusion}

We have analyzed the available observational data on the pulsar PSR B 1706-44 and the SNR G 343.1-2.3 and suggested that these objects could be the remnants of a SN which exploded within a mushroom-like cavity created by the SN progenitor wind breaking out of the parent molecular cloud. 
This accounts for the disparity between the measured and implied velocities of the pulsar. Our suggestion implies that in addition to the known bright "half" of the SNR G 343.1-2.3 there should exist a more extended and weaker component, so that the actual shape of G343.1-2.3 is similar to that of the wellknown SNR VRO 42.05.01. We have found such a component in archival radio data. Further observations, such as those discussed in Sect. 2.2, would be useful to confirm or reject the association between this component and the SNR G 343.1-2.3.

Acknowledgements. We are grateful to R. Dodson for providing his manuscript in advance of publication, and to the referee (J. M. Cordes) for several useful suggestions. V. V. Gvaramadze is supported in part by the Deutscher Akademischer Austausch Dienst (DAAD).

\section{References}

Arzoumanian, Z., Chernoff, D. F., \& Cordes, J. M. 2002, ApJ, 568, 289

Becker, W., Brazier, K. T. S., \& Trümper, J. 1995, A\&A, 298, 528

Blaauw, A., \& Ramachandran, R. 1998, J. Astrophys. Astron., 19, 19

Blandford, R. D., \& Romani, R. W. 1988, MNRAS, 234, 57P

Camilo, F. 1996, in Pulsars: Problems and Progress, ed. S. Johnston, M. A. Walker, \& M. Bailes (San Francisco: ASP), ASP Conf. Ser., 105,39

Chevalier, R. A., \& Liang, E. P. 1989, ApJ, 344, 332

Ciotti, L., \& D'Ercole, A. 1989, A\&A, 215, 347

Clark, D. H., \& Caswell, J. L. 1976, MNRAS, 174, 267

Cordes, J. M., \& Rickett, B. J. 1998, ApJ, 507, 846

Dodson, R., Golap, K., Osborne, J., UdayaShankar, N., \& Rao, A. P. 2001, in The Universe at Low Radio Frequencies, ed. A. Pramesh Rao (San Francisco: ASP), IAU Symp., 199, in press

Duncan, A. R., Stewart, R. T., Haynes, R. F, \& Jones, K. L. 1995, MNRAS, 277, 36

Frail, D. A., Goss, W. M., \& Whiteoak, J. B. Z. 1994a, ApJ, 437, 781

Frail, D. A., Kassim, N. E., \& Weiler, K. W. 1994b, AJ, 107, 1120
Franco, J., Tenorio-Tagle, G., Bodenheimer, P., \& Różyczka, M. 1991, PASP, 103, 803

Gaensler, B. M., \& Johnston, S. 1995, Proc. Astron. Soc. Aust., 12, 76

Giacani, E. B., Frail, D. A., Goss, W. M., \& Vieytes, M. 2001, AJ, 121,3133

Gorenstein, P., \& Tucker, W. H. 1976, ARA\&A, 14, 373

Green, D. A. 1991, PASP, 103, 209

Gupta, Y. 1995, ApJ, 451, 717

Gupta, Y., Rickett, B. J., \& Lyne, A. G. 1994, MNRAS, 269, 1035

Gvaramadze, V. V. 1999a, A\&A, 352, 712

Gvaramadze, V. V. 1999b, in Astrophysics on the Boundary of Centuries, ed. N. S. Kardashev, R. D. Dagkesamansky, \& Yu. A. Kovalev (Moscow: ASC), 163 (in Russian)

Gvaramadze, V. V. 2001a, A\&A, 374, 259

Gvaramadze, V. V. 2001b, A\&A, 369, 174

Gvaramadze, V. V. 2002, in Neutron Stars in Supernova Remnants, ed. P. O. Slane, \& B. M. Gaensler (San Francisco: ASP), ASP Conf. Ser., 271, 23

Huang, Z. P., Thuan, T. X., Chevalier, R. A., Condon, J. J., \& Yin, Q. F. 1994, ApJ, 424, 114

Johnston, S., Lyne, A. G., Manchester, R. N., et al. 1992, MNRAS, 255, 401

Johnston, S., Nicastro, L., \& Koribalski, B. 1998, MNRAS, 297, 108

Kaspi, V. M. 1996, in Pulsars: Problems and Progress, ed. S. Johnston, M. A. Walker, \& M. Bailes (San Francisco: ASP), ASP Conf. Ser., 105,375

Koribalski, B., Johnston, S., Weisberg, J. M., \& Wilson, W. 1995, ApJ, 441,756

Lorimer, D. R., Bailes, M., \& Harrison, P. A. 1997, MNRAS, 289, 592

Lozinskaya, T. A. 1992, Supernovae and Stellar Winds in the Interstellar Medium (New York: AIP)

McAdam, W. B., Osborne, J. L., \& Parkinson, M. L. 1993, Nature, 361,516

Nicastro, L., Johnston, S., \& Koribalski, B. 1996, A\&A, 306, L9

Pineault, S., Landecker, T. L., \& Routledge, D. 1987, ApJ, 315, 580

Taylor, J. H., \& Cordes, J. M. 1993, ApJ, 411, 674

Thompson, R. J., \& Córdova, F. A. 1994, ApJ, 421, L13 